\title{
CHECKLIST DAS ESPÉCIES DE EUPHORBIACEAE JUSS. OCORRENTES NO SEMI-ÁRIDO PERNAMBUCANO, BRASIL ${ }^{1}$
}

\author{
Marccus Vinícius Alves ${ }^{2}$
}

Recebido em 22/01/1999. Aceito em 27/07/1999

\begin{abstract}
RESUMO - (Checklist das espécies de Euphorbiaceae Juss. ocorrentes no semi-árido pernambucano, Brasil) É apresentado o check-list das Euphorbiaceae da região semi-árida do Estado de Pernambuco (zona da Caatinga, subzonas do Agreste e do Sertão), nordeste do Brasil. Foram estudados os materiais oriundos das coletas realizadas durante os anos de 1997-1999 e aqueles depositados nos Herbários locais (IPA, PEUFR, TASH e UFP). Foram registradas 89 espécies, distribuídas em 22 gêneros, sendo Croton L., Cnidoscolus Pohl, Dalechampia Plum. ex L., Euphorbia L. e Phyllanthus L. os melhor representados, com 21, 7, 6, 6 e 6 táxons, respectivamente. Caperonia castanefolia A. St.Hil, Euphorbia phosphorea Mart. e Romanoa tamnoides (A. Juss.) Radcl.-Sm. são novas citaçōes para a flora pernambucana.
\end{abstract}

Palavras-chave - Euphorbiaceae, semiárido, Pernambuco, Brasil

\begin{abstract}
Checklist of Euphorbiaceae Juss. of the semi-arid in Pernambuco State, Brazil). This paper presents a checklist of Euphorbiaceae of the semi-arid area - Caatinga zone, subzones Agreste and Sertão - in Pernambuco State (Northeast of Brazil). Materials from field work done from 1997 to 1999 and from the local Herbaria (IPA, PEUFR, TASH and UFP), were studied. Up to now, 89 species, distributed within 22 genera have been listed. Croton L. (21), Cnidoscolus Pohl (7), Dalechampia Plum. ex L. (6), Euphorbia L. (6) and Phyllanthus L. (6), were the most important genera as regards number of species. Caperonia castanefolia A. St.-Hil, Euphorbia phosphorea Mart. and Romanoa tamnoides (A. Juss.) Radcl.-Sm. are new records for Pernambuco State.
\end{abstract}

Key words - Euphorbiaceae, Semi-arid, Pernambuco State, Brazil

\section{Introdução}

Euphorbiaceae Juss. é a única família de Euphorbiales que inclui representantes nativos (Cronquist 1981). Diversos autores (Pax 1890; Hutchinson 1969; Webster 1974; 1987; 1994; Meeuse 1990) consideram as Euphorbiaceae como uma das maiores famílias de Angiospermas, abrangendo cerca de 7.800 espécies, distribuídas por 300 gêneros em 5 subfamílias. De acordo com Cronquist (1981), são plantas que ocorrem preferencialmente em ambientes tropicais e subtropicais, havendo, no entanto,

\footnotetext{
1 Auxílio FACEPE

2 Departamento de Botânica, CCB, Universidade Federal de Pernambuco, Av. Moraes Rego s.n., CEP 50670-901, Recife, Pernambuco, Brasil.e-mail: malves@ smtp-gw.ibot.sp.gov.br
} 
representantes em regiões com temperaturas mais amenas, sejam elas em decorrência da latitude ou da altitude.

Segundo Barroso et al. (1981), as Euphorbiaceae estão presentes nos diferentes ecossistemas brasileiros com cerca de 1.100 espécies agrupadas em 72 gêneros. Barbosa et al. (1996), tendo por base informações bibliográficas, indicam cerca de 500 espécies ocorrentes no nordeste do Brasil, das quais grande parte em área de Caatinga.

Apesar do número expressivo de espécies da família na área de abrangência do semi-árido nordestino, são raros os tratamentos florísticos ou taxonômicos sobre o grupo. Em Pernambuco mais especificamente, Gallindo (1985) dedicou-se a análise taxonômica do gênero Jatropha L. e, mais recentemente, Lucena (1996) e Webster et al. (1998) estudaram as espécies de Euphorbiaceae ocorrentes em alguns dos Brejos de Altitude. Silva (1997) por sua vez, realizou abordagem sobre os óleos essenciais das sementes de algumas espécies da família com distribuição na Caatinga local. Silva (1989) aportou dados sobre a anatomia de Jatropha mutabilis (Pohl) Baill., espécie muito comum no semi-árido do Estado.

No entanto, ainda são poucos os dados disponíveis sobre o grupo na região, que além de mostrar-se representativo na flora da Caatinga (Lima 1957; Sales et al. 1998) apresenta inúmeras espécies com amplo uso popular (medicinal, madeireiro e ornamental).

Assim, busca-se contribuir para o melhor conhecimento das Euphorbiaceae, através de check-list das espécies catalogadas para o limite do semi-árido no Estado de Pernambuco e viabilizar futuros estudos com a família.

\section{Material e métodos}

Foi realizado levantamento do acervo botânico dos Herbários: IPA (Instituto de Pesquisas Agronômicas), PEUFR (Universidade Federal Rural de Pernambuco), TASH (EMBRAPA - Centro de Pesquisas do Trópico Seco, Petrolina) e UFP (Universidade Federal de Pernambuco), onde foram relacionados os espécimes coletados no semiárido pernambucano (zona da Caatinga, subzonas do Agreste e do Sertão), segundo a delimitação fitofisionômica proposta por Lima (1957).

Paralelamente, foram realizadas excursões para coleta de material botânico em diversos municípios das subzonas do Agreste e do Sertão do Estado de Pernambuco, durante o período de janeiro/1997 a fevereiro/1999. O material coletado foi incluído no acervo do Herbário UFP, com duplicatas no IPA e no PEUFR.

Para as identificações foram empregadas referências específicas (Alves 1993; 1994; Cordeiro 1992; 1995; Gallindo 1985; Lima 1989; Lucena 1996; Müller 1874; Rogers \& Appan 1973; Secco 1997; Smith 1988; Webster \& Armbruster 1991), além da análise comparativa com materiais de várias Instituições e interpretação de fotos de tipos existentes no Laboratório de Sistemática de Fanerógamas, Departamento de Botânica, Universidade Federal de Pernambuco.

Acompanhando o nome específico, segue-se, quando existente, o nome popular empregado na região de estudo, além dos materiais de referência. Para a distribuição geográfica no Estado de Pernambuco estão indicadas as microrregiões geográficas onde foram coletadas as exsicatas estudadas. 
Com relação às microrregiões geográficas estabelecidas para o Estado de Pernambuco (Guizzo et al. 1992) foram adotadas as seguintes abreviaturas: ARA Araripina, SAL - Salgueiro, SPSF - Sertão Pernambucano do São Francisco, APJ - Alto Pajeú, SMX - Sertão do Moxotó, ARC - Arcoverde, ASP - Agreste Setentrional Pernambucano, VIP - Vale do Ipojuca, AMP - Agreste Meridional Pernambucano, MSP - Mata Seca Pernambucana, REC - Recife e MUP - Mata Úmida Pernambucana (Fig. 1).

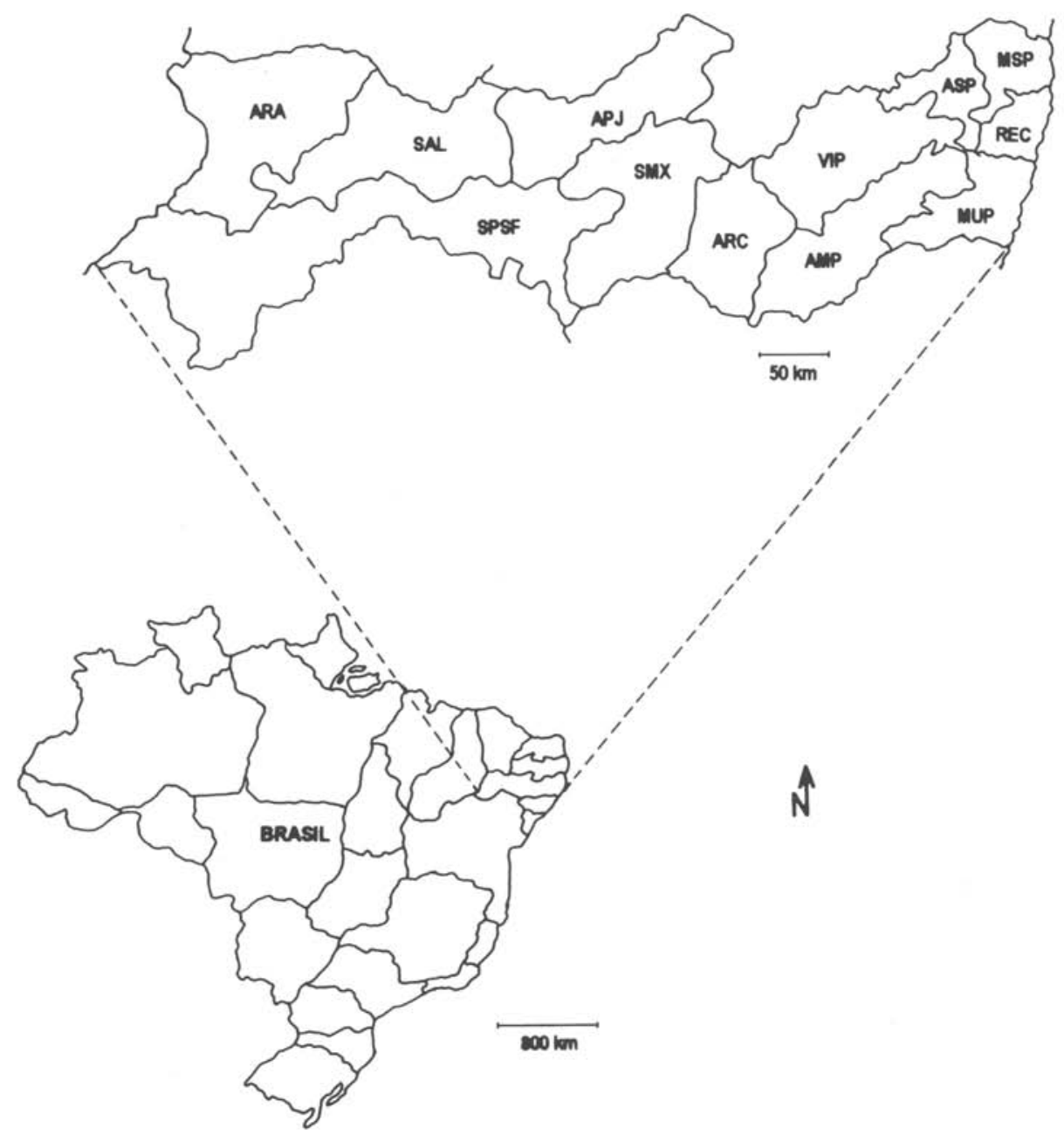

Figura 1. Estado de Pernambuco e as microrregiões geográficas. ARA - Araripina; SAL - Salgueiro; SPSF - Sertão Pernambucano do São Francisco; APJ - Alto Pajeú; SMX - Sertão do Moxotó; ARC - Arcoverde; ASP - Agreste Setentrional Pernambucano; VIP - Vale do Ipojuca; AMP - Agreste Meridional Pernambucano; MSP - Mata Seca Pernambucana; REC - Recife; MUP - Mata Úmida Pernambucana. 
ARA, SAL, SPSF, APJ, SMX e ARC incluem-se na subzona fitogeográfica do Sertão e ASP, VIP e AMP na subzona do Agreste. MSP, REC e MUP estão, em quase sua totalidade, fora dos limites do semi-árido pernambucano (zonas da Mata e Litoral). Porém, em algumas áreas do extremo oeste destas microrregiões podem ser encontrados alguns trechos com vegetação de Caatinga.

Área de Estudo - O Estado de Pernambuco, situado na região equatorial, no nordeste brasileiro, ocupa superfície de $98.107 \mathrm{~km}^{2}$. A pluviosidade é elevada no litoral (em torno de $2.000 \mathrm{~mm}$ anuais) e nas serras, enquanto que nas demais áreas a média raramente ultrapassa $800 \mathrm{~mm}$ anuais e as chuvas distribuem-se de maneira irregular ao longo do ano. A natureza errática das chuvas no semi-árido nordestino é uma das principais características observadas nos índices pluviométricos locais (Sampaio 1995).

A Serra da Borborema ( $1.300 \mathrm{~m}$ de altitude) atravessa o Estado de norte a sul e representa o limite biogeográfico entre as áreas de vegetação xerófila e úmida. As serras interioranas e os "chapadões" são constituídos por afloramentos cretáceos e graníticos, apresentando índices pluviométricos mais elevados do que o entorno imediato.

Lima $(1957 ; 1981)$ dividiu o Estado em quatro zonas fitofisionômicas: Litoral, Mata, Savana e Caatinga. Esta última, que representa cerca de $80 \%$ do Estado, abrange as subzonas do Agreste e do Sertão, possuindo características do clima semi-árido, como os baixos e irregulares índices pluviométricos acompanhados de fortíssima insolação. O Agreste (caatinga hipoxerófila) diferencia-se pelas baixas altitudes, pluviosidade relativamente alta, solos em geral profundos e vegetação levemente adensada. O Sertão (caatinga hiperxerófila) por sua vez, compartilha com o Agreste as baixas altitudes, porém apresenta geralmente solos pouco profundos, índices pluviométricos mais baixos e vegetação menos densa. Os Brejos de Altitudes (Lima 1957) ocorrem tanto no Agreste quanto no Sertão e distinguem-se destes pela altitude acima de $1.000 \mathrm{~m}$, solos profundos, índices pluviométricos elevados e pela vegetação arbórea de grande porte, formando núcleos florestais dentro do limite do semi-árido.

Famílias como Leguminosae, Euphorbiaceae e Cactaceae, além de Malvaceae, Bromeliaceae, Convolvulaceae, Asteraceae e Poaceae são citadas freqüentemente para diversos trechos da Caatinga pernambucana como os principais componentes da paisagem (Araújo et al. 1995; Lima 1957; 1981; Rodal 1984).

Para o presente trabalho limitou-se a área de estudo ao semi-árido do Estado de Pernambuco, não incluindo, portanto, os Brejos de Altitude uma vez que estes apresentam maiores similaridades florísticas com a vegetação da zona da Mata do que com a da Caatinga (Lima 1957; Sales et al. 1998). No entanto, as espécies presentes nas áreas de contato entre a vegetação dos Brejos de Altitute e da Caatinga foram registradas.

\section{Resultados e discussão}

O semi-árido nordestino é, provavelmente, uma das regiões brasileiras de maior diversidade em espécies da família Euphorbiaceae. Ao final desse estudo foram registradas 89 espécies distribuídas em 22 gêneros (Tab. 1). Deste total, Croton L. é o mais representativo, com 21 espécies (aproximadamente 1/4 das catalogadas). Outros gêneros porém, apesar de menos diversificados, são particularmente importantes pelo 
Tabela 1. Relação das espécies de Euphorbiaceae Juss. nativas do semi-árido pernambucano.

Acalypha brasiliensis Müll. Arg.

Ocorrência: ARA, APJ, VIP, AMP

Materiais de referência: Correia 167 et al. (UFP), Heringer et al. 554 (PEUFR)

A. multicaulis Müll. Arg.

Ocorrência: ARA, SAL, SPSF, APJ, SMX, ARC, ASP, VIP

Materiais de Referência: Fotius 3768 (IPA), Miranda 744 et al. (PEUFR)

A. poiretii Spreng.

Ocorrência: ARA, APJ, SMX

Materiais de referência: Heringer 656 et al. (IPA), Lima 274 et al. (IPA)

A. pruriens Nees \& Mart.

Ocorrência: SPSF, VIP

Materiais de referência: Melo 70 et al. (PEUFR), Silva 86 (PEUFR)

Alchornea castanaefolia A. Juss. ("pau-mole")

Ocorrência: SPSF

Materiais de referência: Andrade-Lima 50-549 (IPA, UFP)

A. triplinervia (Spreng.) Müll. Arg. var. triplinervia

Ocorrência: AMP

Material de referência: Andrade-Lima 70-5674 (IPA)

Bernardia sidoides Müll. Arg. ("maniçobinha")

Ocorrência: ARA, SAL, SPSF, SMX, ARC, VIP, AMP

Materiais de referência: Alves et al. EBNN 1872 (UFP), Fotius 3823 (TASH)

B. tamanduana (Baill.) Müll. Arg.

Ocorrência: APJ, ARC, VIP

Materiais de referência: Ferraz \& Santos 39 (PEUFR), Tenório 66-126 (IPA)

Caperonia castaneaefolia A. St.-Hil.

Ocorrência: ARA, SPSF

Materiais de referência: Alves et al. EBNN 1452 (IPA,UFP), Silva s.n. (TASH 1270)

Chamaesyce chamaeclade (L.) Millsp.

Ocorrência: SPSF, AMP

Materiais de referência: Chiappeta s.n., 02.X.85 (IPA), Fotius 3362 (IPA)

C. hirta (DC) Moldenke ("quebra-pedra")

Ocorrência: ARA, SPSF, ARC, VIP

Materiais de referência: Alves et al. EBNN 1500 (IPA,UFP), Miranda 833 et al. (PEUFR)

C. prostrata (Aiton) Small

Ocorrência: ARA, SPSF, ARC, VIP

Materiais de referência: Alves et al. EBNN 1450 (IPA,UFP), Alves et al. EBNN 1434 (UFP)

C. selloi (Klotzsch \& Garcke) Croizat

Ocorrência: ARA, SAL, SPSF, APJ

Materiais de referência: Alves et al. EBNN 1477 (IPA, UFP), Alves et al. EBNN 1530 (IPA)

C. thymifolia (L.) Millsp. ("mama-de-cabra")

Ocorrência: SAL, SPSF, APJ, ARC, ASP, VIP, AMP

Materiais de referência: Alves et al. EBNN 1698 (IPA,UFP), Silva 552 (PEUFR)

Cnidoscolus bahianus (Ule) Pax \& Hoffm. ("faveleira")

Ocorrência: ARA, SAL, SPSF, APJ

Materiais de referência: Araújo 11 (IPA), Barreto 171 (IPA)

C. ferax Müll. Arg.

Ocorrência: ARA

Material de referência: Lima et al. 45 (IPA)

C. hamosus Pohl

Ocorrência: SPSF, SMX, VIP

Materiais de referência: Alves et al. EBNN 1445 (IPA, UFP), Gouveia et al. 21 (PEUFR)

C. obtusifolius Pohl ("urtiga-de-raposa")

Ocorrência: SPSF, SMX, MSP

Materiais de referência: Gallindo et al. 417 (IPA), Miranda 1214 et al. (PEUFR) 
Tabela 1 (continuação). Relação das espécies de Euphorbiaceae Juss. nativas do semi-árido pernambucano.

C. quercifolius Pohl ("favela-de-cachorro, faveleira") Ocorrência: ARA, SAL, SPAF, APJ, SMX, ARC, VIP

Materiais de referência: Alves et al. EBNN 1491 (IPA,UFP), Alves et al. EBNN 1532 (UFP)

C. urens (L.) Arthur ("cansanção")

Ocorrência: ARA, SAL, SPAF, APJ, SMX, ARC, ASP, VIP, AMP

Materiais de referência: Alves et al. EBNN 1146 (IPA,UFP), Alves et al. EBNN 1538 (UFP)

C. vitifolius Müll. Arg. ("favela-de-galinha")

Ocorrência: ARA, SAL, SPAF, APJ, SMX, ARC, VIP

Materiais de referência: Andrade-Lima 75-8092 (IPA), Gallindo et al. 718 (IPA)

Croton adamantinus Müll. Arg. ("marmeleiro-preto")

Ocorrência: SPSF, SMX, ARC

Materiais de referência: Alves et al. EBNN 1442 (IPA, UFP), Fotius 3827 (TASH)

C. argyrophylloides Müll. Arg. ("sacatinga")

Ocorrência: ARA, SAL, SPSF, APJ, SMX, VIP, MSP

Materiais de referência: Pereira et al. 711 (IPA), Pickel 1469 (IPA)

C. campestris A. St.-Hil. ("velame-branco")

Ocorrência: SPSF, APJ, SMX, ARC, VIP, AMP

Materiais de referência: Alves et al. 1556 (IPA,UFP), Ataide 670 (IPA)

C. comosus Müll. Arg.

Ocorrência: VIP

Material de referência: Andrade-Lima 81-9017 (IPA)

C. floribundus Spreng. ("capixingui”)

Ocorrência: ASP, VIP

Materiais de referência: Lucena et al. 127 (PEUFR), Rodal 657 (PEUFR)

C. glandulosus L. ("gervão")

Ocorrência: ARA, SPSF, ARC, VIP, AMP

Materiais de referência: Alves et al. EBNN 1555 (IPA,UFP), Fotius 3877 (IPA)

C. grewioides Baill.

Ocorrência: SPSF, ARC, VIP

Materiais de referência: Andrade-Lima 66-4866 (IPA), Rodal 54 (IPA)

C. hirtus L'Her.

Ocorrência: SAL, APJ, SMX, VIP

Materiais de referência: Belo \& Guedes 1703 (IPA), Heringer 846 (IPA)

C. lobatus L. ("mato-de-raposa")

Ocorrência: ARA, SAL, SPSF, SMX, VIP

Materiais de referência: Alves et al. EBNN 1492 (IPA,UFP), Alves et al. EBNN 1495 (UFP)

C. luetzelburgii Pax \& Hoffm.

Ocorrência: ARA

Materiais de referência: Fotius 3793 (IPA)

C. micans Sw. emend. Müll. Arg. ("sacatinga, marmeleiro-branco")

Ocorrência: SAL, SPSF, SMX, ARC, VIP, AMP

Materiais de referência: Alves et al. EBNN 1443 (IPA,UFP), Alves et al. EBNN 1451 (UFP)

C. moritibensis Baill.

Ocorrência: SPSF, APJ, ARC, VIP, AMP

Materiais de referência: Fotius 3601 (IPA), Zickel 05 et al. (PEUFR)

C. pulegioides Baill.

Ocorrência: SPSF, VIP

Materiais de referência: Sales 561 et al. (PEUFR), Silva 366 (PEUFR)

C. regnelianus Müll. Arg. ("marmeleiro-bravo")

Ocorrência: SAL, SPSF, APJ, VMX, ARC, VIP

Materiais de referência: Gomes 11 (IPA), Pickel 3535 (IPA)

C. rhamnifolius (Kunth) Müll. Arg. ("velame")

Ocorrência: ARA, SAL, SPSF, APJ, SMX, ARC, ASP, VIP

Materiais de referência: Alves et al. EBNN 1463 (IPA,UFP), Alves et al. EBNN 1494 (UFP) 
Tabela 1. Relação das espécies de Euphorbiaceae Juss. nativas do semi-árido pernambucano.

C. sonderianus Müll. Arg. ("marmeleiro")

Ocorrência: ARA, SAL, SPSF, APJ, SMX, ARC, VIP

Materiais de referência: Alves et al. EBNN 1431 (IPA,UFP), Alves et al. EBNN 1537 (UFP)

C. tricolor Müll. Arg.

Ocorrência: SAL, APJ

Materiais de referência: Fotius 3764 (TASH), Lima \& Gallindo 68 (IPA)

C. tridentatus Müll. Arg.

Ocorrência: SPSF

Materiais de referência: Fotius 3328 (IPA), Fotius 3548 (TASH)

C. urticaefolius Lam.

Ocorrência: SPSF, APJ, SMX, VIP

Materiais de referência: Alves et al. EBNN 1460 (IPA,UFP), Alves et al. EBNN 1465 (UFP)

C. versicolor Müll. Arg.

Ocorrência: ARA

Material de referência: Fotius 3344 (IPA)

C. zehntneri Pax \& Hoffm. ("quebra-faca")

Ocorrência: SAL, SPSF, AMP

Materiais de referência: Araújo 11 (IPA), Fotius 3328 (IPA)

Dalechampia brasiliensis Lam.

Ocorrência: SPSF, VIP

Materiais de referência: Miranda 1688 et al. (PEUFR), Tschá 165 (PEUFR)

D. clausseniana Baill.

Ocorrência: VIP, AMP

Materiais de referência: Alves et al. PROBIO 27 (IPA, UFP), Rodal 456 et al. (PEUFR)

D. ficifolia Lam.

Ocorrência: AMP, MUP

Materiais de referência: Andrade-Lima 67-4978 (IPA), Andrade-Lima 70-5664 (IPA)

D. ilheotica Wawra

Ocorrência: AMP

Material de referência: Félix s.n. , 07.X.1997 (UFP)

D. pernambucensis Baill.

Ocorrência: SPSF, VIP

Materiais de referência: Miranda 1231 et al. (PEUFR), Webster 25635 et al. (IPA)

D. scandens L. ("tamiarana")

Ocorrência: ARA, SPSF, APJ, ARC, VIP, AMP

Materiais de referência: Alves et al. EBNN 1458 (UFP), Webster 25641 et al. (PEUFR)

D. schenckiana Pax \& Hoffm.

Ocorrência: VIP, AMP

Materiais de referência: Silva 235 (PEUFR), Webster 25648 et al. (IPA, neotypus)

Ditaxis desertorum (Müll. Arg.) Pax \& Hoffm.

Ocorrência: ARC

Material de referência: Krapovickas 38049 et al. (IPA)

D. malpighiacea (Ule) Pax \& Hoffm. ("pau-de-macaco")

Ocorrência: ARA, SAL, SPSF, APJ, ARC, VIP

Materiais de referência: Araújo 183 (IPA, PEUFR), Barreto CFPE 169 (IPA, PEUFR)

Euphorbia comosa Vell. ("burra-leiteira")

Ocorrência: ARA, SAL, SPSF, APJ, SMX, VIP, AMP

Materiais de referência: Alves et al. EBNN 1556 (IPA,UFP), Mariz 555 (UFP)

E. goyazensis Boiss.

Ocorrência: ARC

Materiais de referência: Andrade-Lima 75-8063 (IPA), Andrade-Lima 75-8094 (IPA)

E. heterophylla $\mathrm{L}$. ("parece-mas-não-é")

Ocorrência: SPSF, ARC, VIP, AMP

Materiais de referência: Alves et al. EBNN 1496 (UFP, IPA), Tavares 742 (UFP) 
Tabela 1. Relação das espécies de Euphorbiaceae Juss. nativas do semi-árido pernambucano.

E. insulana Vell. ("maleiteira")

Ocorrência: SPSF, APJ, SMX, ASP, VIP, AMP

Materiais de referência: Alves et al. PROBIO 13 (UFP), Alves et al. PROBIO 49 (UFP)

E. phosphorea Mart. ("barbasco")

Ocorrência: SPSF

Materiais de referência: Fotius 3930 (IPA, TASH)

E. sarcodes Boiss.

Ocorrência: ARC

Material de referência: Correia \& Souza 203 (UFP)

Gymnanthes concolor Müll. Arg.

Ocorrência: AMP, MSP

Materiais de referência: Andrade-Lima 54-1910 (IPA), Andrade-Lima 67-4907 (IPA)

Jatropha gossypifolia L. ("pinhão-roxo")

Ocorrência: SAL, SPSF, ARC, VIP

Materiais de referência: Alves et al. EBNN 1448 (IPA,UFP), Alves et al. 1453 (UFP)

J. molissima (Pohl) Baill. ("pinhão-bravo")

Ocorrência: ARA, SAL, SPSF, APJ, SMX, ARC, ASP

Materiais de referência: Alves et al. EBNN 1427 (IPA,UFP), Alves et al. EBNN 1450 (UFP)

J. mutabilis (Pohl) Baill. ("pinhão-miúdo")

Ocorrência: SAL, SPSF, APJ, SMX, ARC

Materiais de referência: Alves et al. EBNN 1438 (IPA, UFP), Carauta 997 (PEUFR)

J. ribifolia (Pohl) Baill. ("pinhão-manso")

Ocorrência: SAL, SPSF, APJ, SMX, ARC, VIP

Materiais de referência: Alves et al. EBNN 1434 (IPA,UFP), Alves et al. EBNN 1476 (UFP)
Mabea occidentalis Benth.

Ocorrência: VIP

Material de referência: Andrade-Lima 71-6594 (IPA)

Manihot caerulescens Pohl emend Rogers \& Appan ("maniçoba-ceará)

Ocorrência: ARA, VIP

Materiais de referência: Alves et al. PROBIO 35 (UFP), Sousa s.n., 13.IV.1986 (UFP)

M. dichotoma Ule ("maniçoba")

Ocorrência: ASP, SPSF, VIP

Materiais de referência: Guedes 115 (IPA), Pickergill et al. RU72-17 (IPA)

M. epruinosa Pax \& Hoffm. ("maniçoba")

Ocorrência: SPSF, APJ, SMX, VIP

Materiais de referência: Alves et al. EBNN 1470 (IPA, PEUFR, UFP), Gomes 43 (IPA)

M. heptaphylla Ule ("maniçoba")

Ocorrência: ARC, VIP

Materiais de referência: Alves et al. EBNN 1457 (IPA,UFP), Griz 40 (UFP)

Maprounea guyanensis Aubl. ("pau-de-leite")

Ocorrência: ARA, SAL, SPSF, APJ, SMX, VIP

Materiais de referência: Guedes 163 (IPA), Ramos 33 (PEUFR)

Pera glabrata (Schott.) Baill. ("pau-leiteiro")

Ocorrência: ASP

Material de referência: Andrade-Lima s.n., 17.X.1949 (IPA)

$P$. heteranthera (Schrank) I.Irtn.

Ocorrência: ASP

Material de referência: Andrade-Lima 54-1910 (IPA)

Phyllanthus acuminatus Vahl

Ocorrência: VIP

Materiais de referência: Lucena 132 et al. (PEUFR), Oliveira 236 et al. (PEUFR)

$P$. carolinensis Walt.

Ocorrência: SPSF

Material de referência: Fotius 3367 (IPA) 
Tabela 1. Relação das espécies de Euphorbiaceae Juss. nativas do semi-árido pernambucano.

P. claussenii Müll. Arg.

Ocorrência: VIP

Materiais de referência: Alves et al. PROBIO 62 (UFP,IPA), Mayo 137 et al. (PEUFR)

P. klotzschianus Müll. Arg.

Ocorrência: VIP, ASP

Materiais de referência: Andrade-Lima 60-3523 (IPA), Pontual 77-1337 (PEUFR)

P. niruri L. ("quebra-pedra-macho")

Ocorrência: ARA, SAL, SPSF, APJ, SMX, ARC, VIP

Materiais de referência: Alves et al. EBNN 1434 (IPA,PEUFR,UFP), Lima 292 (TASH)

P. orbiculatus Rich. emend. Müll. Arg.

Ocorrência: ARA, SAL, SPSF, SMX, ARC, VIP

Materiais de referência: Ataide 149 (IPA), Heringer 155 (IPA)

Romanoa tamnoides (A. Juss.) Radcl.-Sm.

Ocorrência: VIP

Material de referência: Andrade-Lima 70-5922 (IPA)

Sapium glandulatum (Vell.) Pax ("burra-leiteira")

Ocorrência: ARA, SPSF, APJ, SMX, ASP, VIP, AMP

Materiais de referência: Alves 402 (UFP), Ferraz 253 et al. (PEUFR)

$S$. montevidense Klotzsch ex Baill.

Ocorrência: SAL, SPSF, APJ, SMX

Materiais de referência: Guedes 1600 (PEUFR), Fotius 3203 (IPA, TASH)

S. scleratum Ridl.

Ocorrência: ASP

Material de referência: Belo s.n., 07.II.1990 (PEUFR)

Sebastiania brasiliensis Spreng.

Ocorrência: ARA, SAL, SPSF, APJ, SMX, ARC, VIP

Materiais de referência: Andrade-Lima 50-726 (IPA), Tschá 525 (PEUFR)

S. corniculata (Vahl) Müll. Arg.

Ocorrência: SAL, SPSF, SMX

Materiais de referência: Ataide 20 (IPA), Fotius 3824 (TASH)

S. glandulosa (Mart.) Pax

Ocorrência: ARC

Materiais de referência: Andrade-Lima 75-8059 (IPA), Miranda 1733 et al. (PEUFR)

S. hispida (Mart.) Pax

Ocorrência: ARA, ARC

Materiais de referência: Mariz 538 (UFP), Pereira 720 et al. (IPA)

S. marginata Müll. Arg.

Ocorrência: SMX, ARC

Materiais de referência: Ferraz 39 (IPA), Krapovickas 38051 et al. (IPA)

Stillingia trapezoidea Ule

Ocorrência: SPSF, ARC, VIP, AMP

Materiais de referência: Alves et al. EBNN 1148 (IPA,UFP), Correia 447 (PEUFR)

Tragia pohlii Müll. Arg.

Ocorrência: APJ, SMX

Materiais de referência: Andrade-Lima 91 (IPA), Magalhães 4805 (IPA)

T. sellowiana (Klotzsch) Müll. Arg. ("cipó-urtiguinha”)

Ocorrência: SPSF, APJ

Materiais de referência: Heringer 955 et al. (IPA), Lima \& Gallindo 131 (IPA)

T. volubilis L ("cipó-urtiga")

Ocorrência: ARA, SAL, SPSF, APJ, SMX, ARC, ASP, VIP, MSP

Materiais de referência: Alves et al. EBNN 1551 (IPA,PEUFR,UFP), Pickel 2901 (IPA) 
número de espécies registradas - Cnidoscolus Pohl (7 spp.), Euphorbia L. (6 spp.), Dalechampia Plum. ex L. (6 spp.) e Phyllanthus L. (6 spp.).

Silva (1998) registrou a ocorrência de 33 espécies (11 gêneros) de Euphorbiaceae para a zona da Caatinga dos Estados de Pernambuco e Paraíba. Destas, apenas Croton siderophyllus Baill. (SMX), Dalechampia leandri Baill. (SMX) e Chamaesyce oftalmica Perl. (VIP) não se encontram na listagem aqui apresentada. Vale mencionar que algumas das espécies aqui tratadas são novas citações para o Estado de Pernambuco, dentre elas Caperonia castaneaefolia, Euphorbia phosphorea e Romanoa tamnoides.

Evidenciou-se também, para algumas delas, ampla distribuição geográfica no semi-árido do Estado de Pernambuco, como é o caso de Croton rhamnifolius, Croton sonderianus e Jatropha molissima. Estas três espécies, além de estarem melhor representadas nos acervos dos Herbários consultados, ocorrem abundantemente nas diferentes regiões visitadas.

Em contraposição ao acima exposto, foram encontradas espécies que, apesar de ocorrerem em outros Estados brasileiros, apresentam em Pernambuco baixa incidência. Caperonia castaneaefolia, Croton adamantinus, Croton luetzelburgii e Euphorbia sarcodes entre outras, são bons exemplos.

Outras espécies merecem destaque por ocorrerem nas áreas de contato (ecótonos) entre a vegetação de Caatinga e dos Brejos de Altitude. Neste caso, pode-se mencionar Alchornea triplinervia var. triplinervia, Croton comosus, Croton floribundus, Dalechampia clausseniana, Dalechampia ficifolia, Dalechampia ilheotica, Gymnanthes concolor, Mabea occidentalis, Maprounea guyanensis, Pera glabrata e Pera heteranthera.

Aleurites molucana ("nogueira") e Euphorbia tirucalli ("avelós") apesar de não serem nativas devem ser citadas por terem sido observadas e coletadas em formações vegetais naturais na região de estudo. $\mathrm{O}$ mesmo caso se aplica a Manihot esculenta ("mandioca"), espécie nativa que se encontra amplamente cultivada para consumo humano.

Constatou-se que as microrregiões SPSF e VIP são aquelas em que estão registrados o maior número de espécies - 55 e 51, respectivamente. Possivelmente estes valores sejam conseqüência de esforços de coleta concentrados em determinadas áreas do Estado de Pernambuco durante os últimos anos.

\section{Agradecimentos}

O Autor agradece aos curadores dos Herbários consultados; à FACEPE, pelo apoio financeiro concedido; aos revisores, pelas valiosas sugestões; e a todos aqueles que direta ou indiretamente contribuíram para a realização deste trabalho.

\section{Referências bibliográficas}

Alves, M. 1993. Notas sobre o gênero Pera Mutis (Euphorbiaceae). Anales Jardim Botanico de Madrid 5(1): 151-153.

Alves, M. 1994. Notas sobre algumas espécies de Croton L. (Euphorbiaceae) do Brasil. Fontqueria 39: 45-47. Araújo, E.; Sampaio, E. \& Rodal. M. J. N. 1995. Composição florística e fitossociologia de três áreas de caatinga de Pernambuco. Revista Brasileira de Biologia 55(4): 595-607. 
Barbosa, M.; Mayo, S.; Castro, A .; Freitas, G.; Pereira, M.; Gadelha-Neto, P. \& Moreira, H. 1996. Checklist preliminar das Angiospermas. Pp. 253-414. In: E. Sampaio (Eds.), Pesquisa botânica nordestina: progressos e perspectivas. SBB. Recife.

Barroso, G. M.; Guimarães, E.; Ichaso, E.; Costa, C. \& Peixoto, A . L. 1981. Sistemática de Angiospermas do Brasil. vol. 2. Universidade Federal de Viçosa, Viçosa.

Cordeiro, I. 1992. Flora da Serra do Cipó. Minas Gerais: Euphorbiaceae. Boletim de Botânica da Universidade de São Paulo 13: 169-217.

Cordeiro, I. 1995. Euphorbiaceae. Pp. 300-317. In: B. Stannard (Ed.), Flora of the Pico das Almas. Kew, Surrey.

Cronquist, A. 1981. An integrated system of classification of flowering plants. Houghton Mifflin, Boston.

Gallindo, F. 1985. O gênero Jatropha L. (Euphorbiaceae) no Estado de Pernambuco. Dissertação de Mestrado. Universidade Federal Rural de Pernambuco, Recife.

Guizzo, J.; Riva, L. \& Kuhnen, R. 1992. Trabalhando com mapas: Pernambuco. Ática, São Paulo.

Hutchinson, J. 1969. Tribalism in the family Euphorbiaceae. Amererican Journal of Botany 56(7): 738758.

Lima, D. 1957. Estudos fitogeográficos de Pernambuco. Publicaçōes do IPA 2: 1-41.

Lima, D. 1981. The Caatinga dominum. Revista Brasileira de Ciências 4: 149-163.

Lima, D. 1989. Plantas da Caatinga. Academia Brasileira de Ciências, Rio de Janeiro.

Lucena, M. 1996. Levantamento florístico das Euphorbiaceae dos brejos de altitude do Estado de Pernambuco. Monografia de Bacharelado. Universidade Federal Rural de Pernambuco, Recife.

Meeuse, A. 1990. The Euphorbiaceae auct. plur., an unnatural taxon. Euburon, Kopenhagen.

Müeller, C. 1874. Euphorbiaceae. In. C. Martius (Ed.), Flora Brasiliensis 11(2): 1-568.

Rodal, M. 1984. Fitoecologia de uma área do médio Vale do Moxotó, Pernambuco. Dissertação de Mestrado. Universidade Federal Rural de Pernambuco, Recife.

Rogers, R. \& Appan, S. 1973. Manihot, Manihotoides (Euphorbiaceae). Flora Neotropica 13: 1-272.

Pax, F. 1890. Euphorbiaceae. In: A. Engler \& K. Prantl (Eds.), Die Naturlichen Pflanzenfamilien III3(5): 1-119.

Sales, M.; Rodal, M. J. N. \& Mayo, S. 1998. Plantas vasculares das florestas serranas de Pernambuco. Um check-list da flora ameaçada dos brejos de altitude. Pernambuco, Brasil. Universidade Federal de Pernambuco, Recife.

Sampaio, E. 1995. Overview of the Brazilian Caatinga. Pp.35-63. In: S. Bullock \& R. Davidse (Eds.), Seasonally dry tropical forest. Cambridge University Press, Cambridge.

Secco, R. 1997. Revisão taxonômica das espécies neotropicais da tribo Alchorneae (Hurusawa) Hutchinson (Euphorbiaceae). Tese de Doutorado. Universidade de São Paulo, São Paulo.

Silva, C. 1989. Morfologia e estrutura interna comparativa de espécimens distintos de Jatropha mutabilis (Pohl) Baill. (Euphorbiaceae). Dissertação de Mestrado. Universidade Federal Rural de Pernambuco, Recife.

Silva, S. Euphorbiaceae da Caatinga: distribuição das espécies e potencial oleaginoso. Tese de Doutorado. Universidade de São Paulo, São Paulo.

Smith, L.; Downs, R. \& Klein, R. 1988. Euphorbiaceae. Pp. 1-408. R. Reitz (Ed.), Flora Ilustrada Catarinense.

Webster, G. 1974. Conspectus of a new classification of the Euphorbiaceae. Taxon 24: 593-601.

Webster, G. 1987. The saga of the spurges: a review of classification and relationships in the Euphorbiales. Botanical Journal of the Linnean Society 94: 3-46.

Webster, G. 1994. Synopsis of the genera and suprageneric taxa of Euphorbiaceae. Annals of the Missouri Botanical Garden 81(1): 33-144.

Webster, G. \& Armbruster, W. 1991. A synopsis of the neotropical species of Dalechampia (Euphorbiaceae). Botanical Journal of the Linnean Society 105: 137-77. 